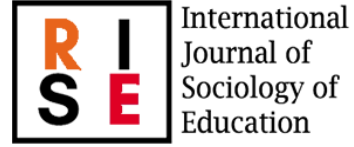

International

Journal of

Sociology of

Education

\section{Hipatia Press}

www.hipatiapress.com

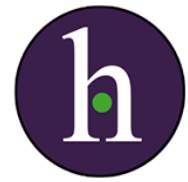

Instructions for authors, subscriptions and further details:

http://rise.hipatiapress.com

\title{
Las Interacciones con el Voluntariado en los Grupos Interactivos y el Éxito Escolar
}

Juan García López ${ }^{1}$

Sandra López Fernández ${ }^{2}$

$M^{a}{ }^{a}$ Carmen Sánchez Pérez ${ }^{2}$

1) Subred Universitaria de Comunidades de Aprendizaje de Castilla-La Mancha, Spain

2) Universidad de Castilla-La Mancha

Date of publication: February $25^{\text {th }}, 2021$

Edition period: February 2021-June 2021

To cite this article: García López, J., López Fernández, S., \& Sánchez Pérez, M.C. (2021). Las Interacciones con el Voluntariado en los Grupos Interactivos y el Éxito Escolar. International Journal of Sociology of Education, 10(1), 29-56. http://doi.org/10.17583/rise.2021.6043

To link this article: http://dx.doi.org/10.17583/rise.2021.6043

PLEASE SCROLL DOWN FOR ARTICLE

The terms and conditions of use are related to the Open Journal System and to Creative Commons Attribution License (CC-BY) 


\section{Volunteers' Interactions through Interactive Groups and Educational Success}

Juan García López

Subred Universitaria de

Comunidades de Aprendizaje de

Castilla-La Mancha
Sandra López Fernández

Universidad de Castilla La Mancha

$\mathrm{M}^{\mathrm{a}}$ Carmen Sánchez Pérez

Universidad de Castilla La Mancha

(Received: 28 June 2020; Accepted: 30 September 2020; Published: 25 February 2021)

\section{Abstract}

This paper aims to analyze Language and Mathematics performance improvement when Interactive Groups are held at school. This study was developed applying a communicative methodology and gathered information through interviews, focus groups and data analysis. It provides evidence on how interactions with volunteers involved in the classroom foster the students' educational success in the schools studied. Results, in line with similar studies, point out that holding Interactive Groups at school is linked to an academic achievement improvement for those groups and students involved. Evidence point out the relevant role of participation in the educative process. Interactions provided by volunteers' involvement, their diversity and the intensity linked to this Successful Educational practice, are key elements for the students' educational success and the improvement process.

Keywords: interactive groups, educational success, volunteering, interactions, learning communities. 


\section{Las Interacciones con el Voluntariado en los Grupos Interactivos y el Éxito Escolar}

Juan García López

Subred Universitaria de

Comunidades de Aprendizaje de

Castilla-La Mancha
Sandra López Fernández

Universidad de Castilla La Mancha

$\mathrm{M}^{\mathrm{a}}$ Carmen Sánchez Pérez

Universidad de Castilla La Mancha

(Recibido: 15 Junio 2020; Aceptado: 30 Septiembre 2020; Publicado: 25 Febrero 2021)

\section{Resumen}

El objetivo de este artículo es analizar si el rendimiento escolar en las áreas de aprendizaje de Lenguaje y Matemáticas mejora tras la organización del aula en Grupos interactivos. El estudio, realizado a través de la metodología comunicativa, mediante entrevistas, grupos de discusión y análisis de datos secundarios, aporta evidencias de cómo las interacciones que se producen en el aula con la participación del voluntariado han mejorado el éxito escolar del alumnado en los centros educativos analizados. Los resultados, en línea con estudios previos, muestran que la organización de Grupos interactivos en el aula ofrece una mejora de los resultados académicos en los grupos de alumnos donde se llevan a cabo. Las evidencias apuntan al importante papel que desempeña la participación en el proceso educativo. Las interacciones con el voluntariado, su heterogeneidad y la intensidad de esta práctica son elementos esenciales en el proceso de mejora y en el éxito escolar del alumnado

Palabras clave: grupos interactivos, éxito escolar, voluntariado, interacciones, comunidades de aprendizaje 
as interacciones y la participación de la comunidad son dos de los factores más importantes en el aprendizaje dialógico de la sociedad actual y en la implantación de las Actuaciones Educativas de Éxito, tal como señalan las teorías internacionales más referenciadas (Aubert et al., 2008) y muestran numerosas investigaciones (García y DíezPalomar, 2015; Oliver y Gatt, 2010). La información disponible en el ámbito de los centros de Comunidades de Aprendizaje de Castilla-La Mancha en este sentido es, sin embargo, escasa. Con el objetivo de recabar datos, en colaboración y coordinación con los centros, sobre la aplicación de las Actuaciones Educativas de Éxito, la Subred Universitaria de Comunidades de Aprendizaje de Castilla-La Mancha desarrolló una investigación, referida al curso 2017-2018 (Sánchez y García, 2019)². Entre las evidencias aportadas por este estudio, el papel de los Grupos interactivos en la mejora de los resultados de aprendizaje del alumnado, la intensidad en su implantación y el papel del voluntariado dentro del aula (Vieira y Puigdellívol, 2013), son objeto de atención en este artículo. El estudio que aquí se presenta realiza contribuciones relevantes a esta línea de investigación, al presentar evidencias sobre el éxito académico vinculado al desarrollo de Grupos interactivos en el aula.

Los objetivos del estudio se orientaron, por un lado, a conocer si los colegios analizados mejoraron el rendimiento escolar en el curso 2017-18, tras la implantación de los Grupos interactivos. Así mismo se buscaba analizar si el desarrollo de esta Actuación Educativa de Éxito se había llevado a cabo con la suficiente intensidad para facilitar la aceleración de los aprendizajes. Finalmente, se estudió si los colegios cumplían con el criterio de la participación de un voluntariado diverso en los Grupos interactivos, que propiciase interacciones transformadoras.

Así, tras presentar las bases teóricas que fundamentan el estudio, se analizan y debaten las evidencias recogidas en los colegios de Comunidades de Aprendizaje de Castilla-La Mancha seleccionados. Se mostrará la mejora producida en los resultados escolares de los niños y niñas de estos centros. Cabe concluir, por tanto, la importancia de la aplicación de Grupos interactivos y la participación del voluntariado en la mejora y transformación socioeducativa de los centros. 


\section{García López, López Fernández, \& Sánchez Pérez}

\section{Los Grupos Interactivos Generan Éxito escolar}

En las Comunidades de Aprendizaje se desarrollan Actuaciones Educativas de Éxito bajo los principios del aprendizaje dialógico. La participación de la comunidad en el aula, a través del voluntariado, es un principio fundamental: familiares, antiguos alumnos y alumnas, estudiantes universitarios, entidades sociales del entorno, etc. (Elboj et al, 2002). Abrir las aulas al entorno social del que se nutren las niñas y los niños supone incrementar las interacciones, produciendo una mejora en el aprendizaje. Los Grupos interactivos se caracterizan por ser transferibles a cualquier contexto educativo y social; se implantan para la transformación educativa y social de la comunidad (DíezPalomar y Flecha, 2010). Las evidencias indican que, entre otros logros, se trata de una práctica de éxito para la superación del fracaso y el abandono escolar (Racionero et al., 2012). Instituciones internacionales, como la Comisión Europea, se han hecho eco de ello, recomendando la transformación de centros educativos en Comunidades de Aprendizaje como estrategia para reducir el abandono escolar temprano y aumentar los resultados académicos, mejorando la convivencia y el desarrollo de valores (European Commission, 2011).

Un aspecto clave para implementar los Grupos interactivos es el carácter heterogéneo de la agrupación del alumnado (Valls et al., 2011) y del voluntariado, que puede ser un familiar, un estudiante universitario, otro profesor o figuras sociales del entorno (Valls y Kyriakides, 2013). Se conseguirá así generar un contexto enriquecedor, con otros agentes, que hará que las interrelaciones en el aula produzcan efectos de mejora y favorezcan la conexión del alumnado con el mundo (Bruner, 1997, p. 38).

La heterogeneidad será, pues, un elemento fundamental, entendiéndose a todos los niveles: cultural, étnica, social e incluso, económicamente. Se trata de traer al aula los recursos necesarios para que niños y niñas alcancen un nivel educativo con las máximas expectativas (Elboj y Gràcia, 2005, p. 105).

Así, los Grupos interactivos se han configurado como la forma de organización de aula que consigue mejores resultados, tanto en el aprendizaje como en la convivencia (Valls et al., 2016, p. 2). Otras Actuaciones Educativas de Éxito también evidencian la mejora de resultados: bibliotecas 
tutorizadas (Aguilera et al., 2010), tertulias dialógicas (Aubert y García, 2001), formación de familiares (Jasis y Ordoñez-Jasis, 2012) y actuaciones educativas en prevención de violencia de género (Pulido et al., 2014).

En este estudio se pondrá el énfasis en los Grupos interactivos como la Actuación Educativa de Éxito con capacidad de mejorar el rendimiento escolar del alumnado.

\section{Interacciones más Intensas con el Voluntariado en el Aula}

El voluntario o voluntaria asignado a cada grupo se convierte en una nueva posibilidad de interacción, un nuevo elemento en el proceso de aprendizaje del alumnado. La interacción con otras personas aumenta y mejora los resultados del proceso, al tiempo que tiene la capacidad de transformar el contexto. Abrir las aulas a la sociedad tiene como consecuencia la transformación de las mismas. No solo se observan los efectos positivos en el centro educativo, también mejoran las relaciones con el profesorado y las familias, trasladándose el contexto educativo al entorno social que lo envuelve, resultando un clima más solidario en la comunidad (Flecha y Soler, 2013).

En la práctica de los Grupos interactivos, el profesorado plantea la resolución de una actividad de forma colaborativa entre sus miembros. El papel del docente aquí se modifica: ya no es el elemento guía en la realización de la actividad, sino que supervisará las tareas encomendadas a cada grupo y la interacción que se producirá entre el grupo heterogéneo y el voluntariado. El papel fundamental de este último es la dinamización del grupo, favoreciendo las relaciones entre todos sus miembros y fomentando el apoyo solidario. El profesorado es responsable del desarrollo de los contenidos que se van a trabajar: materiales, tiempos, asignación de grupos, así como del análisis de los resultados (Aubert y García, 2001). Mantendrá una relación de ayuda mutua con el voluntariado, resolviendo la actividad propuesta y facilitando la asistencia que este solicite (Racionero et al., 2009). El voluntariado, a su vez, debe motivar al grupo de forma generalizada, sin olvidar a aquellos niños y niñas que pueden quedarse fuera del trabajo conjunto.

Se concluye de esto que la participación del voluntariado es primordial en 


\section{García López, López Fernández, \& Sánchez Pérez}

la transformación de un centro educativo. En ningún caso debemos entender esta presencia en el aula como la sustitución del personal docente, sino como un elemento diferenciador para la consecución del mayor número de interacciones con el alumnado, que tiene como resultado un espacio enriquecido de solidaridad y cooperación (Sordé et al., 2014; Vieria, 2010). No se trata de poner en práctica acciones aisladas con validez científica cuestionada, sino que la transformación de un colegio en Comunidades de Aprendizaje supone la puesta en marcha de actuaciones que tienen los mejores resultados para la superación del fracaso escolar y la calidad de los centros (INCLUD-ED Consortium, 2011).

En el análisis desarrollado en este estudio se evidencia que, en centros educativos transformados en Comunidades de Aprendizaje, donde los Grupos interactivos y las interacciones intensas han formado parte del proceso de aprendizaje, se observa una mejora sustancial en los resultados escolares finales de su alumnado.

\section{Voluntariado, Nueva Clave del Aprendizaje en la Sociedad de la Información}

Es imposible entender la importancia del rol del voluntariado en el aprendizaje sin ubicarlo en el actual contexto social. En la sociedad de la información, el aprendizaje del alumnado ya no depende exclusivamente de lo que ocurre dentro del aula. En esta nueva situación se transforma el acceso a la información, y la vida de los alumnos está continuamente estimulada por su entorno (Castells, 1996). La educación debe contemplar esta interacción constante y compensar el peso del contexto familiar, a veces, nada positivo.

La sociedad de la información ha traído importantes novedades en las teorías de las ciencias sociales y educativas: los aspectos dialógicos aparecen en primera línea del debate sobre la acción social. La concepción comunicativa de la acción social (Habermas, 1987) ha planteado como el aprendizaje depende principalmente de las interacciones entre personas. Bajo esta nueva concepción, la práctica del aprendizaje dialógico plantea importantes transformaciones en la forma de enseñar y de aprender, para mejorar el éxito escolar de todo el alumnado, atendiendo a la igualdad de diferencias. 
El rol del voluntariado estará guiado por los siete principios fundamentales del aprendizaje dialógico: diálogo igualitario, dimensión instrumental, inteligencia cultural, transformación, creación de sentido, solidaridad e igualdad de diferencias (Aubert et al., 2008). Bajo estos principios, se conseguirá mejorar la convivencia de las diversas realidades que se incluyen en el escenario del aula, así como el rendimiento escolar (Racionero et al., 2012). A través del voluntariado, se fundamenta la concepción dialógica del aprendizaje, siendo un agente idóneo para la estimulación constante, que potencie el desarrollo del alumnado (Mercer y Howe, 2012).

El aprendizaje dialógico y sus principios, llevados al aula a través de los Grupos interactivos, son elementos necesarios en el proceso de enseñanza en la sociedad de la información. En una sociedad donde la rapidez y la inmediatez son características primordiales, las Comunidades de Aprendizaje responden a estas demandas sociales. Se trata, en resumen, de apostar por la creencia de que todo el alumnado es capaz de conseguir éxitos, bajo el compromiso y cooperación de voluntariado y profesorado (Elboj et al., 1998).

\section{Aceleración de Aprendizajes con el Voluntariado en el Aula}

En el voluntariado se asienta el valor añadido de acelerar los aprendizajes, de incorporar otra realidad al aula. Se trata de personas que no necesariamente poseen un perfil académico y, por tanto, aportan al contexto del aula la percepción de una realidad externa a la misma, creando así interacciones diversas, acercando el entorno y acelerando el aprendizaje (González y Moll, 2002). El voluntariado enriquece los Grupos interactivos con un capital cultural (Bourdieu, 1986), independientemente de su nivel académico, ya que todos disponemos de lenguaje y argumentación (Habermas, 1987). Su presencia en el aula fomenta el espacio compartido entre alumnado y personas adultas, además de reducir las ratios y conseguir un proceso de enseñanza concentrado en el grupo (Herrero y Brown, 2010).

Diversas investigaciones, y esta también, inciden en la importancia de la participación de familiares y voluntariado en relación con el bienestar del alumnado y el rendimiento escolar resultante (Jeynes, 2003). Sheldon (2003) evidenció que la implicación de la familia y la comunidad en el aprendizaje del alumnado tenían un efecto directo y positivo en los niveles académicos. 


\section{García López, López Fernández, \& Sánchez Pérez}

Otras investigaciones, anteriores a la conceptualización de las Actuaciones Educativas de Éxito, en los años noventa, ya constataron la importancia de la cultura comunitaria, destacando su importante papel en el aprendizaje (Nettles y Greenberg, 1990; Sánchez, 1999).

Otras líneas de investigación, que tienen como objeto el estudio del voluntariado en la escuela, se orientan a analizar los programas comunitarios en los que las familias actúan como voluntarios (Deslandes, 2006; Valls y Kyriakides, 2013; Wanat, 2010). La investigación INCLUD-ED, del VI Programa Marco de la Unión Europea (INCLUD-ED Consortium, 2011), tras el análisis de resultados, concluía que se produjo un incremento en torno al $70 \%$ de alumnos que alcanzaban las competencias básicas después de cinco años aplicando Grupos interactivos. Son recientes los estudios que muestran que, tras la aplicación de Grupos interactivos, se ha conseguido una mayor implicación del alumnado, ha crecido el compromiso adquirido para la consecución de la tarea, observando una mejora en la convivencia (Chocarro y Sáenz, 2016). Se comprueba también que la presencia del voluntariado en el aula es un valor añadido a lo aprendido dentro de esta y enriquece las interacciones (Álvarez y Puigdellívol, 2014). Así mismo, se han constatado efectos positivos en la inclusión del alumnado tras la puesta en marcha de los Grupos interactivos, resultando relevantes los procesos de planificación de las actividades y formación del voluntariado para aumentar su participación (Muntaner et al., 2015).

El voluntariado incorpora a la escuela la esencia del contexto cultural: saberes de la vida cotidiana, costumbres, tradiciones, valores; la comunidad en forma de capital cultural (González y Moll, 2002). Favorece la relación entre escuela y entorno, sobre todo en contextos de grupos sociales vulnerables (Díez et al., 2011). El conocimiento se genera en estas situaciones de interacción entre las diferentes personas que, en este caso, conforman el aula. El aprendizaje que resulta de esta interrelación no solo amplía el conocimiento de aquellos a los que les es dirigido, sino que a su vez está modificando el contexto social (Aubert et al., 2008).

\section{Metodología}

El presente trabajo parte de la metodología generada en el estudio elaborado 
por la Subred Universitaria de Comunidades de Aprendizaje de Castilla-La Mancha (Sánchez y García, 2019), que recabó con los centros de Comunidades de Aprendizaje información, entre otras cuestiones, sobre la implantación de los Grupos interactivos y el papel del voluntariado. La recogida de datos se realizó en el tercer trimestre del curso 2017-2018, siguiendo los planteamientos de la investigación comunicativa crítica (Gómez et al., 2006), a través de técnicas cualitativas y cuantitativas: entrevistas, grupos de discusión con miembros de la comunidad y análisis de documentos (actas de las sesiones de trabajo de las Comisiones mixtas, memorias de los centros educativos y registros de sesiones de trabajo). De la información recogida serán objeto de análisis en este trabajo las variables: presencia y perfil del voluntariado e intensidad de la práctica de los Grupos interactivos.

Cabe destacar que la intensidad en la implantación de los Grupos interactivos es considerada un factor determinante en la capacidad de acelerar el ritmo de los aprendizajes (Elboj et al., 2005; Flecha, 2015). En este estudio se conceptualizó a través de cuatro indicadores:

1. Porcentaje de grupos-clase de los colegios que organizaban regularmente Grupos interactivos.

2. Número de sesiones de trabajo de Grupos interactivos que el colegio realizó en todas las áreas de aprendizaje del currículo a lo largo del tercer trimestre del curso 2017-2018.

3. Periodicidad de la práctica de los Grupos interactivos (según la escala: semanal, quincenal, ocasionalmente)

4. Heterogeneidad del alumnado en los Grupos interactivos.

En cuanto al análisis de las fuentes secundarias, se ha explotado la base de datos de la administración autonómica Delphos ${ }^{2}$, relativa al rendimiento escolar. Este se ha conceptualizado como los resultados académicos obtenidos por el alumnado de los seis cursos de Educación Primaria en las evaluaciones finales de curso escolar, en las áreas de aprendizaje de Lenguaje y Matemáticas, tal y como el profesorado lo registró en la citada base de datos.

El indicador utilizado ha sido "rendimiento del colegio", obtenido a partir de las calificaciones individuales de los alumnos (y de cada grupo-clase), según la escala de $\operatorname{logros}^{3}$ de 1 a 10 puntos. Para evaluar los progresos, se han considerado los datos de los cursos 2016-17 y 2017-18.

La muestra considerada para este estudio está constituida por cuatro de los 


\section{García López, López Fernández, \& Sánchez Pérez}

diez colegios de Comunidades de Aprendizaje de Castilla-La Mancha reconocidos por la administración educativa regional en ese momento. Estos centros presentan situaciones de origen heterogéneas, pero comparten la necesidad de mejora en el éxito del alumnado (Tabla 1). Para la selección de la muestra se utilizaron criterios acordes con los objetivos que este estudio aborda: centros que evidenciaban progresos en el rendimiento escolar a lo largo del curso 2017-2018, que contaban con un número suficiente de voluntariado y de perfiles diversos para implantar los Grupos interactivos y que mostraban suficiente intensidad en la implantación de los Grupos interactivos a través de los indicadores definidos. 


\section{Tabla 1. \\ Características de los colegios de la muestra \\ - Localidad: Puertollano. \\ - Provincia: Ciudad Real. \\ - Ubicación: separado del resto de la ciudad. Próximo a viviendas de Colegio Doctor promoción pública con características de gueto urbano y social. \\ Limón - Perfil del alumnado: mayoría de etnia gitana. \\ - Características: elevado absentismo, alto nivel de fracaso escolar, conductas disruptivas que rompen la convivencia, falta de motivación por el aprendizaje, escasa participación familiar, asistencia irregular.}

\begin{tabular}{|c|c|}
\hline Colegio La Paz & $\begin{array}{l}\text { - Localidad: Albacete. } \\
\text { Provincia: Albacete. } \\
\text { Ubicación: colegio situado en un barrio desfavorecido a las afueras de la } \\
\text { ciudad en una zona de exclusión. } \\
\text { Perfil del alumnado: representativo del barrio, en el que conviven tres } \\
\text { tipos de población: aproximadamente la mitad es paya, un tercio de etnia } \\
\text { gitana y el resto, población inmigrante. } \\
\text { Características: centro desfavorecido y excluido, donde el abandono y el } \\
\text { fracaso escolar es alto. Sus resultados han ido mejorando lentamente con } \\
\text { la transformación en Comunidades de Aprendizaje desde el curso 2006- } \\
07\end{array}$ \\
\hline Colegio Pío XII & $\begin{array}{l}\text { - Localidad: Ciudad Real. } \\
\text { Provincia: Ciudad Real. } \\
\text { Ubicación: ha sufrido un proceso de deterioro hasta llegar a una situación } \\
\text { que se califica de gueto. } \\
\text { Perfil del alumnado: mayoría de etnia gitana. } \\
\text { Características: centro pequeño, con pérdida de matrícula. Partía de una } \\
\text { situación con problemas de convivencia en las aulas, absentismo escolar } \\
\text { y una baja participación de los familiares. El profesorado piensa que la } \\
\text { mejor educación es la que se ofrece desde el respeto y el reconocimiento } \\
\text { de las minorías, de las diferencias y las diversidades. }\end{array}$ \\
\hline $\begin{array}{l}\text { Colegio S. Juan de } \\
\text { Ávila }\end{array}$ & $\begin{array}{l}\text { - } \quad \text { Localidad: Castellar de Santiago. } \\
\text { Provincia: Ciudad Real. } \\
\text { - } \quad \text { Ubicación: Colegio único de población rural. } \\
\text { Perfil del alumnado: No tiene problemas de absentismo ni abandono } \\
\text { escolar. Las familias tienen un nivel económico medio-bajo y muestran } \\
\text { un alto grado de implicación en la vida del centro. } \\
\text { Características: La búsqueda de un modelo más inclusivo, la mejora de } \\
\text { los resultados escolares y de la convivencia, junto con la intención de } \\
\text { transformación del entorno social y cultural, motivaron la creación de la } \\
\text { Comunidades de Aprendizaje en el curso 2010-11. }\end{array}$ \\
\hline
\end{tabular}

\section{Elaboración propia a partir de informaciones aportadas por los Centros.}

\section{Como se aprecia, los colegios de la muestra presentan en unos casos}




\section{García López, López Fernández, \& Sánchez Pérez}

situaciones desfavorecidas de gueto y en otros, una aspiración a la mejora de expectativas. En todos, el trasfondo de la necesidad de cambiar un modelo reproductor de prácticas educativas y estructuras sociales segregadoras que venían desarrollando, hacia un modelo transformador igualitario (Aubert et al., 2009; García et al., 2013). Se aspiraba a la transformación social y educativa de los centros y su entorno, para evitar la exclusión de los niños y niñas y conseguir así mejores resultados en el proceso de aprendizaje.

En cuanto al perfil de las personas entrevistadas y participantes en los grupos de discusión (Tabla 2), fue diverso y representativo del conjunto de la comunidad.

Tabla 2.

Perfiles personales de las Entrevistas y de los Grupos de discusión Entrevistas Grupos de discusión

\begin{tabular}{|c|c|c|}
\hline Doctor Limón & $\begin{array}{l}\text { Equipo directivo, tutores y tutoras de } 1^{\circ} \\
\text { de Educación Infantil, } 2^{\circ}, 3^{\circ}, 5^{\circ} \text { y } 6^{\circ} \text { de } \\
\text { Educación Primaria }\end{array}$ & 1 miembro del Equipo directivo \\
\hline La Paz & $\begin{array}{l}\text { Equipo directivo, tutores y tutoras de } \\
1^{\circ}, 2^{\circ} \text { y } 3^{\circ} \text { de Educación Infantil, y } 4^{\circ} \\
\text { de Educación Primaria }\end{array}$ & $\begin{array}{l}1 \text { docente del Claustro } \\
1 \text { miembro de la Asociación de } \\
\text { Familiares de Alumnos }\end{array}$ \\
\hline Pío XII & $\begin{array}{l}\text { Equipo directivo, tutores y tutoras de } \\
1^{\circ}, 2^{\circ}, 5^{\circ} \text { y } 6^{\circ} \text { de Educación Primaria }\end{array}$ & $\begin{array}{l}1 \text { representante de las entidades } \\
\text { colaboradoras }\end{array}$ \\
\hline $\begin{array}{l}\text { San Juan de } \\
\text { Ávila }\end{array}$ & $\begin{array}{l}\text { Equipo directivo, tutores y tutoras de } \\
1^{\mathrm{o}}, 2^{\mathrm{o}}, 3^{\mathrm{o}} \text { de Educación infantil y } 1^{\mathrm{o}}, 2^{\mathrm{o}} \text {, } \\
3^{\mathrm{o}} 4^{\mathrm{o}}, 5^{\mathrm{o}}, 6^{\mathrm{o}} \text { de Educación primaria }\end{array}$ & 1 representante del alumnado \\
\hline
\end{tabular}

Elaboración propia a partir de informaciones aportadas por los Centros

\section{Presentación y Análisis de Resultados}

Los centros escolares analizados implantaron Comunidades de Aprendizaje con el fin de transformar los colegios y sus entornos. Comprendieron que la mejora del rendimiento escolar dependía de la riqueza de las interacciones que se pudieran introducir en el aula y priorizaron, entre las distintas Actuaciones 
Educativas de Éxito, la implantación de los Grupos interactivos, para acelerar el aprendizaje de los niños y niñas y mejorar el rendimiento escolar.

\section{Rápida Aceleración de los Aprendizajes con los Grupos interactivos}

Numerosas evidencias muestran que los Grupos interactivos son una Actuación Educativa de Éxito reconocida por la comunidad científica internacional como la práctica de aprendizaje, dentro del aula, que presenta los mejores resultados de éxito con todo el alumnado, independientemente del contexto educativo donde se implante (INCLUD-ED Consortium, 2011).

Los resultados del rendimiento escolar obtenidos por el alumnado de los cuatro centros muestran una mejora, en situaciones educativas de fracaso escolar complejas. La Tabla 3 muestra la evolución de los rendimientos escolares del alumnado de los cuatro colegios tras la implantación de esta Actuación Educativa de Éxito.

Tabla 3.

Evolución del rendimiento escolar en las áreas curriculares de Lenguaje y Matemáticas.

\begin{tabular}{|c|c|c|c|c|c|c|}
\hline & \multicolumn{2}{|c|}{$\begin{array}{l}\text { Rendimiento escolar } \\
\text { final del curso 2016- } \\
17\end{array}$} & \multicolumn{2}{|c|}{$\begin{array}{l}\text { Rendimiento escolar } \\
\text { final del curso 2017- } \\
18\end{array}$} & \multicolumn{2}{|c|}{$\begin{array}{c}\text { Incremento } \\
\text { Rendimiento escolar }\end{array}$} \\
\hline & Lenguaje & $\begin{array}{l}\text { Matemá } \\
\text { ticas }\end{array}$ & Lenguaje & $\begin{array}{l}\text { Matemá } \\
\text { ticas }\end{array}$ & Lenguaje & $\begin{array}{c}\text { Matemá } \\
\text { ticas }\end{array}$ \\
\hline Doctor Limón & 4,5 & 4,6 & 5,0 & 4,9 & 0,5 & 0,3 \\
\hline La Paz & 5,2 & 4,7 & 5,3 & 5,5 & 0,1 & 0,8 \\
\hline Pío XII & 3,1 & 2,9 & 4,2 & 3,4 & 1,1 & 0,5 \\
\hline $\begin{array}{l}\text { San Juan de } \\
\text { Ávila }\end{array}$ & 7,1 & 6,7 & 7,3 & 6,9 & 0,2 & 0,2 \\
\hline
\end{tabular}

Fuente: Programa Delphos. Consejería de Educación. JCCM 


\section{García López, López Fernández, \& Sánchez Pérez}

La tarea emprendida para que los propios niños y niñas resuelvan las actividades a través de la ayuda mutua y la solidaridad, en los Grupos interactivos, ha mejorado los resultados escolares. Además, como queda reflejado en la Figura 1, esa mejora se ha producido en todos los colegios.

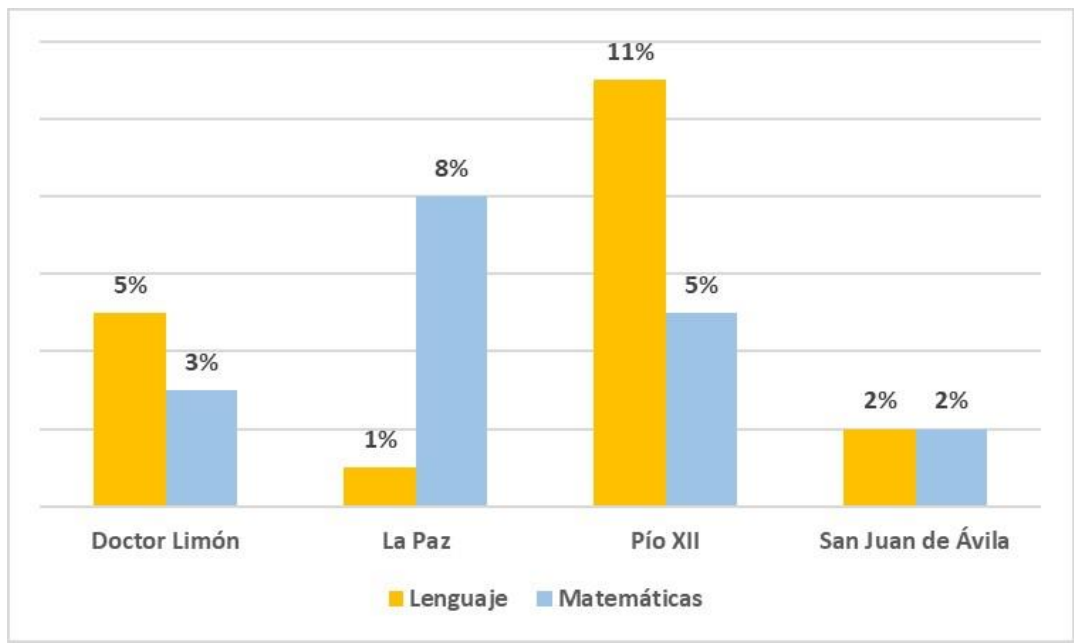

Figura 1. Incremento del rendimiento escolar durante el curso 2017-18. Elaboración propia a partir de datos del estudio.

La puesta en marcha de los Grupos interactivos en el primer año de implantación de Comunidades de Aprendizaje de dos colegios (Dr. Limón y Pío XII), ha permitido superar las situaciones de entornos sociales desfavorecidos en tan solo un año de transformación. También han seguido progresando los otros dos colegios con más experiencia en la implantación de Actuaciones Educativas de Éxito. La Paz (Flecha y Soler, 2013), tras lentos pero sucesivos progresos en varios años, ha incrementado el éxito en Matemáticas un 8\% y mejora sus resultados desde el curso 2006-2007, que inició su transformación. El colegio San Juan de Ávila sigue avanzando en sus expectativas desde el curso 2010-2011. El colegio Pío XII, con una base de éxito baja, ha incrementado el rendimiento escolar en Lenguaje en un $11 \%$.

Los porcentajes de mejora en algunos centros son muy valiosos para el alumnado y la comunidad, pues han supuesto una inflexión en la 
transformación de los colegios y en el deterioro del aprendizaje en los últimos años, mejorando la autoestima de la comunidad y desarrollando altas expectativas. Con estos datos se evidencia que la implantación de los Grupos interactivos supone una mejora constante y paulatina en los resultados académicos.

\section{Aportación de la Diversidad del Voluntariado para enriquecer las Interacciones Transformadoras}

Los centros educativos han obtenido resultados académicos que avalan la mejora tras la implantación de los Grupos interactivos. En el análisis realizado, se evidencia que todos los colegios ajustaron la presencia del voluntariado a las necesidades y perfil del alumnado. Su participación se produce en un número suficiente para posibilitar una organización eficaz de los Grupos interactivos, en una horquilla que oscila entre 11 y 36 personas voluntarias en el tercer trimestre del curso 2017-2018.

El voluntariado conforma un perfil muy diverso, que aporta a la comunidad y a los niños y niñas un rico bagaje sociocultural, que está ayudando a mejorar los rendimientos escolares. En todos los colegios participan familiares de los propios niños y niñas, jóvenes de los barrios, personas de los Centros de mayores, voluntariado de las entidades que colaboran en el proyecto de Comunidades de Aprendizaje, alumnado de las Facultades de Educación realizando el Prácticum de tercer o cuarto curso (Prados y Aguilera, 2013) y alumnado participante en el programa de Aprendizaje Servicio que impulsan profesores y profesoras de las Facultades de Educación (Tabla 4). 


\section{García López, López Fernández, \& Sánchez Pérez}

Tabla 4.

Presencia y perfil del voluntariado en los Grupos interactivos.

Voluntariado Perfil del voluntariado

\begin{tabular}{lcl}
\hline Doctor Limón & 36 & $\begin{array}{l}\text { Alumnado Facultad de Educación, Centro de } \\
\text { mayores y familiares } \\
\text { Voluntariado de las entidades, familiares (con } \\
\text { frecuencia tíos y abuelas), alumnado de la ESO, } \\
\text { exalumnos y alumnado de la F de Educación }\end{array}$ \\
Pío XII & 31 & $\begin{array}{l}\text { Alumnado Facultad de Educación, voluntarios } \\
\text { entidades Secretariado Gitano y familiares }\end{array}$ \\
$\begin{array}{l}\text { San Juan de } \\
\text { Ávila }\end{array}$ & 29 & $\begin{array}{l}\text { Familiares, familiares de antiguos alumnos y } \\
\text { alumnas, antiguo alumnado, voluntariado de } \\
\text { entidades }\end{array}$ \\
\hline
\end{tabular}

Elaboración propia a partir de datos del estudio.

La diversidad del voluntariado para cooperar con los y las profesionales de la educación mejora el contexto de aprendizaje en los Grupos interactivos, posibilitando la aceleración de los aprendizajes en el periodo de un curso escolar. En el caso de los colegios Doctor Limón y Pío XII, en su incorporación a Comunidades de Aprendizaje, y La Paz y San Juan de Ávila con experiencias previas de implantación (véase Tabla 1). Cuando el aula se comparte con personas que no son docentes, con diferentes perfiles, se motiva especialmente al alumnado (Molina, 2011). Se logra el objetivo de alcanzar los máximos resultados escolares del alumnado, aportando una diversidad cultural y cognitiva que asegura el aprendizaje dialógico.

\section{La Intensidad en los Grupos Interactivos para Acelerar los Aprendizajes}

Para que los Grupos interactivos puedan producir el efecto de acelerar los aprendizajes, es necesario que su práctica en el aula tenga la intensidad adecuada, en relación con las características del alumnado. Se ha podido constatar una correlación entre la intensidad en la aplicación de Grupos Interactivos y los resultados (Flecha, 2015).

En este estudio se analizó la intensidad en la implantación de Grupos 
interactivos en los colegios analizados, a través de varios indicadores.

El primer indicador considerado fue el porcentaje de implantación en los grupos-clase: ¿se aplican los Grupos interactivos en todos los grupos-clase o lo hacen parcialmente? La Tabla 5 recoge el nivel de implantación de los Grupos interactivos en todos los colegios del estudio: tres de ellos presentan un desarrollo completo, al $100 \%$ en todos los cursos; el otro se encontraría al $66,6 \%$ (cuatro grupos-clase de los seis que conforman la Educación primaria).

Tabla 5.

Indicadores de intensidad en la implantación de los Grupos interactivos en el $3^{\text {er }}$ trimestre del curso 2017-18.

\begin{tabular}{|c|c|c|c|c|}
\hline & $\begin{array}{c}\% \\
\text { Implantación } \\
\text { en los grupos }\end{array}$ & $\begin{array}{c}\mathrm{N}^{\mathrm{o}} \text { sesiones } \\
3^{\text {er }} \text { trimestre } \\
\text { (1) }\end{array}$ & Periodicidad & $\begin{array}{l}\text { Heterogeneidad } \\
\text { grupos }\end{array}$ \\
\hline Doctor Limón & 100 & 19 & Quincenal & SI \\
\hline $\mathrm{LaPaz}$ & 100 & 20 & $\begin{array}{l}\text { Semanal/ } \\
\text { Quincenal }\end{array}$ & SI \\
\hline Pío XII & 66,6 & 26 & Semanal & SI \\
\hline San Juan de Ávila & 100 & 54 & Semanal & SI \\
\hline
\end{tabular}

Elaboración propia a partir de datos del estudio.

Todos los colegios realizan un número importante de sesiones de trabajo con Grupos interactivos a lo largo del tercer trimestre del curso 2017-18. La franja oscila entre las 19 sesiones del colegio Doctor Limón hasta las 54 del colegio San Juan de Ávila. La periodicidad más habitual en la planificación es la semanal.

Un último indicador fue la heterogeneidad del alumnado en la composición de los Grupos interactivos, como un elemento que incrementa la riqueza de las interacciones de todos los niños y niñas, sin ningún tipo de discriminación. En todos los colegios se tuvo en cuenta la heterogeneidad para la organización de los grupos, tomando en consideración criterios de género, nivel de aprendizaje y origen cultural y religioso. 


\section{García López, López Fernández, \& Sánchez Pérez}

Cabe afirmar, por tanto, que los colegios objeto de estudio tienen unos niveles adecuados en cuanto a la intensidad de implantación de los Grupos interactivos, de acuerdo con los cuatro indicadores considerados (Tabla 5) y las peculiaridades que presentaba el alumnado. El logro del incremento en el rendimiento escolar alcanzado por los cuatro colegios a lo largo del curso 2017-2018, evidencia la importancia de la correcta aplicación de esta Actuación Educativa de Éxito.

\section{Discusión}

Numerosas investigaciones (INCLUD-ED Consortium, 2011; Valls y Kyriakides, 2013) ya han evidenciado que el aprendizaje dialógico es el más adecuado para responder a las nuevas demandas y requerimientos formativos de la sociedad de la información (Elboj et al., 2006). En este estudio se ha comprobado cómo la organización del aula en Grupos interactivos asegura el aprendizaje dialógico y el éxito escolar. A través de las interacciones que se producen con la presencia del voluntariado en el aula, se desarrollan capacidades instrumentales y facilitadoras del procesamiento de la información, necesarias en la sociedad de la información.

En algunos colegios del estudio los incrementos de los resultados (reflejados en la Tabla 3) pueden parecer simplemente discretos. Sin embargo, al tomar en consideración que se trata de variaciones positivas del rendimiento en un solo curso escolar y que uno de los obstáculos de partida era el fracaso escolar, se han cumplido, sin lugar a duda, las expectativas sobre la potencialidad de los Grupos interactivos para mejorar el rendimiento escolar del alumnado. Ante las evidencias aportadas, cabe afirmar que la transformación de estos centros en Comunidades de Aprendizaje ha posibilitado la mejora del éxito escolar con resultados relevantes.

En Comunidades de Aprendizaje, los docentes comparten la gestión educativa y cooperan con diferentes agentes de la comunidad para maximizar el aprendizaje de todo el alumnado. En esta línea de cooperación, la organización de los Grupos interactivos necesita la presencia de una persona adulta en cada grupo dentro del aula. Su papel es el de fomentar interacciones transformadoras necesarias para que los niños y niñas sean capaces de resolver las actividades propuestas por el tutor o tutora, a través de la solidaridad y de 
la ayuda mutua (Aubert et al., 2009). En este estudio, la presencia de suficiente número de voluntarios y voluntarias y con perfiles diversos en los colegios, ha posibilitado los progresos del alumnado hacia la inclusión y el éxito académico.

También se ha podido constatar la aportación del voluntariado a la riqueza e intensidad de las interacciones. Su contribución proviene de la cooperación del profesorado, familiares y voluntariado del entorno. Cuanto más diverso es el perfil del voluntariado (Tabla 4), la práctica resulta más enriquecedora.

Desde el colegio San Juan de Ávila resaltan la aportación del voluntariado y las interacciones en los Grupos interactivos: "La colaboración y apoyo entre alumnos y entre alumnos y voluntarios... se ha apreciado más empatía" (Miembro del Equipo Directivo).

Se han presentado evidencias de cómo los Grupos interactivos aseguran el aprendizaje dialógico y permiten alcanzar las expectativas de la comunidad sobre el éxito escolar del alumnado en un corto plazo de tiempo. La presencia del voluntariado permite a los niños y niñas acelerar sus aprendizajes instrumentales en Lenguaje y Matemáticas (Tabla 3), así como en otros aprendizajes relacionados con las emociones y los valores, como el respeto a todas las culturas que conviven en los cuatro colegios.

Tutores y tutoras del colegio San Juan de Ávila expresan los cambios observados en el aprendizaje en estos términos "Se ha observado una mejora en las relaciones sociales, debido a las distintas estrategias que conlleva el uso del aprendizaje dialógico en el aula..." (Tutora de Educación Infantil). "En relación, a la atención a la diversidad se ha observado un clima de aula que favorece el aprendizaje colaborativo y la inclusión del alumnado con distintas necesidades..." (Tutora de Educación Primaria).

En los colegios analizados, los voluntarios y voluntarias en cada Grupo interactivo motivaron el intercambio de ideas, la ayuda, el apoyo mutuo y el desarrollo cooperativo de la resolución de las actividades planteadas. Todo ello desde la perspectiva del diálogo igualitario y el principio de solidaridad. En los Grupos interactivos se educa en solidaridad y, aunque no se trabaje de una forma conceptual, se practica a diario entre profesorado, voluntariado y alumnado; es una condición básica para conseguir el éxito (De Botton, 2015).

Se conocen evidencias sobre el valor de la heterogeneidad del alumnado (Valls y Kyriakides, 2013) para resolver las actividades propuestas por el tutor 
o tutora, identificando las dificultades desde diferentes perspectivas, y fomentando en el alumnado interacciones transformadoras. Estas facilitan que todos los componentes del grupo puedan alcanzar el éxito en la actividad propuesta sirviéndose de la heterogeneidad que presentan. Se constata que los cuatro colegios estudiados organizaban los Grupos interactivos sin ningún tipo de segregación y teniendo en cuenta la heterogeneidad, atendiendo a criterios de género, nivel de aprendizaje y origen cultural y religioso.

Para todos los colegios, los Grupos interactivos también han supuesto un cambio positivo en el clima de aula y de la convivencia. El colegio Dr. Limón manifiesta: "En los Grupos interactivos destaca la mejoría en la convivencia... se crea en el aula una sesión de trabajo tranquila y un ambiente de motivación..." (Tutora de Educación Primaria). Desde el colegio Pío XII expresan los beneficios en las relaciones intragrupo: "Se aprecia la positiva mejora de las relaciones entre distintos miembros que formaban el grupo de trabajo..." (Tutora de Educación Primaria). También se destaca que se han apreciado mejoras en el aprendizaje: "Los Grupos interactivos han creado un clima agradable, adecuado para el aprendizaje y fomento de la motivación del alumnado..." (Tutora de Educación Primaria).

Para que se produzca la mejora del éxito escolar es necesario que los Grupos interactivos se apliquen con la intensidad necesaria. Al igual que conocemos que en medicina ciertos tratamientos basados en evidencias científicas y aplicados adecuadamente curan las enfermedades, de los Grupos interactivos tenemos suficientes evidencias (Flecha y Soler, 2013; Valls y Kyriakides, 2013) para afirmar que producen efectos en la educación, acelerando los aprendizajes. En este estudio se aprecian evidencias de que los indicadores de intensidad contemplados participan en la aceleración del rendimiento escolar del alumnado.

Los Grupos interactivos acercaron hasta el alumnado la posibilidad de aprender, conociendo otras realidades de su entorno. El voluntariado es, sin duda, un elemento necesario y esencial en la puesta en marcha y consecución de los objetivos que marcan todas las Actuaciones Educativas de Éxito. 


\section{Conclusiones}

Las evidencias ofrecidas por los datos coinciden con la total unanimidad expresada por los colegios, al considerar los Grupos interactivos como una práctica que ha tenido efectos positivos en su transformación como Comunidad de Aprendizaje, en la mejora del aprendizaje y en la convivencia. Los miembros de la comunidad resaltan las altas expectativas que los centros educativos mantienen sobre la transformación de los colegios y la positiva evolución de los rendimientos escolares del alumnado.

Lograr la mejora de los resultados ha sido posible al transformar los colegios en Comunidades de Aprendizaje y seguir las evidencias científicas a través de prácticas que producen igualdad y éxito para todo los niños y niñas. El éxito radica en implantar Actuaciones Educativas de Éxito y aplicarlas con la intensidad necesaria, de acuerdo con las características del alumnado. Los Grupos interactivos se muestran en nuestro estudio como la práctica que produce más éxito en el alumnado, independientemente de las peculiaridades socioeducativas del colegio y su entorno.

Se ha podido constatar que involucrar a los familiares y a la comunidad del entorno para participar como voluntariado en los Grupos interactivos, desde un enfoque dialógico, ha sido un elemento esencial en la transformación de la práctica educativa y ha propiciado la rápida aceleración de los aprendizajes. Los datos recogidos ofrecen evidencias que muestran como el alumnado de los centros ha mejorado su rendimiento escolar en Lenguaje y Matemáticas.

Los Grupos interactivos se manifiestan como una alternativa para la transformación de situaciones de desigualdad educativa. Ofrecen la posibilidad de implantar una educación cargada de diversidad para todos y todas, de forma igualitaria (García y Díez-Palomar, 2015). La práctica de Grupos interactivos tiene como resultado una mejora sustancial en las habilidades sociales, fomentando el trabajo cooperativo con aprendizajes más consolidados, bajo las premisas de la diversidad (Álvarez-Álvarez, 2017).

La heterogeneidad, tanto del alumnado en la organización de los Grupos interactivos, como en la composición del voluntariado, resulta también evidente en el presente artículo (Valls y Kyriakides, 2013; Álvarez y Puigdellívol, 2014). La participación heterogénea del voluntariado en el aula tiene como resultado un mayor número de interacciones y, por lo tanto, mayor 


\section{García López, López Fernández, \& Sánchez Pérez}

posibilidad de éxito, produciendo mejoras en los resultados del proceso enseñanza y aprendizaje (Elboj y Niemelä, 2010).

Cabe dejar constancia de algunas limitaciones del estudio. Sería importante tener la oportunidad de analizar resultados en periodos más amplios de observación y de recogida de datos, para poder confirmar las tendencias y reforzar las conclusiones alcanzadas. Así mismo, sería necesario seguir acumulando evidencias sobre los efectos de la presencia del voluntariado dentro del aula en el proceso de enseñanza y aprendizaje (Vieira y Puigdellívol, 2013), al ser esta una línea de trabajo con enorme potencial.

Dar continuidad a este estudio en años consecutivos permitiría analizar los resultados en relación con la estabilidad de la implantación de los Grupos interactivos y perfilar tendencias. Implicaría desarrollar un estudio longitudinal en relación con la puesta en marcha de los Grupos interactivos en Comunidades de Aprendizaje, un ámbito de estudio aún poco desarrollado en el contexto de Castilla-La Mancha.

Las comunidades de los colegios tenían un sueño: evitar la exclusión de los niños y niñas y mejorar los resultados académicos. Conocer las prácticas de Comunidades de Aprendizaje basadas en evidencias les permitió crear el clima propicio para generar altas expectativas en sus respectivas comunidades. La transformación de los colegios y sus entornos está activada. Los sueños son posibles con evidencias científicas y las Actuaciones Educativas de Éxito.

\section{Notas}

1. Con la colaboración de la Consejería de Educación, Cultura y Deportes de la Junta de Comunidades de Castilla-La Mancha

2. Datos proporcionados por la Consejería de Educación, Cultura y Deporte.

3. Escala de evaluación: Insuficiente (0-4), Suficiente (5), Bien (6), Notable (7-8), Sobresaliente (9-10).

\section{Referencias}

Aguilera, A.; Mendoza, A.; Racionero, S. y Soler, M. (2010). El papel de la universidad en las comunidades de aprendizaje. Revista Interuniversitaria de Formación del Profesorado, 67(24,1), 45-56. 


\section{https://www.redalyc.org/pdf/274/27419180004.pdf}

Álvarez, C. y Puigdellívol, I. (2014). Cuando la comunidad entra en la escuela: un estudio de casos sobre grupos interactivos, valorados por sus protagonistas. Profesorado. Revista de currículum y formación del profesorado, 18(3), 239-253.

http://www.ugr.es/local/recfpro/rev183COL3.pdf

Álvarez-Álvarez, C. (2017). Comunicación, entendimiento y aprendizaje en grupos interactivos. Investigación en la Escuela, 91, 90-105.

http://dx.doi.org/10.12795/IE.2017.i91.06

Aubert, A. y García, C. (2001). Interactividad en el aula. Cuadernos de Pedagogía, 301, 20-24. http://mediasviewer.wkcols.com Aubert, A., Flecha, A., García, C., Flecha, R. y Racionero, S. (2008). Aprendizaje Dialógico en la Sociedad de la Información. Hipatia Editorial.

Bourdieu, P. (1986). The forms of capital. En J.G. Richardson (ed.), Handboodk of Theory and research for the Sociology of Education (pp. 241-258). Greenwood Press.

Bruner, J. S. (1997). La educación, puerta de la cultura. Visor.

Castells, M. (1996). La era de la información: economía, sociedad y cultura (V.1). Alianza.

Chocarro de Luis, E. y Sáenz de Jubera, M. (2016). Grupos interactivos: estrategia para la mejora de la convivencia, la participación y el aprendizaje. Revista Complutense de Educación, 27(2), 585-601. https://doi.org/10.5209/rev_RCED.2016.v27.n2.46911no

De Botton, L. (2015). La dimensión instrumental en las comunidades de aprendizaje. Intangible capital, 11(3), 350-371.

http://dx.doi.org/10.3926/ic.659

Deslandes, R. (2006). Designing and Implementing School, family, and Community Collaboration Programs in Quebec, Canada. The School Community Journal, 16(1), 81-106. https://files.eric.ed.gov/fulltext/EJ794805.pdf

Díez, J., Gatt, S., y Racionero, S. (2011). Placing Immigrant and Minority Family and Community Members at the School's Centre: the role of community participation. European Journal of Education, 46(2), 184196. https://doi.org/10.1111/j.1465-3435.2011.01474.x 


\section{García López, López Fernández, \& Sánchez Pérez}

Díez-Palomar, J., y Flecha, R. (2010). Comunidades de aprendizaje: Un proyecto de transformación social y educativa. Revista interuniversitaria de formación del profesorado, 24(67), 19-30. https://www.redalyc.org/pdf/274/27419180002.pdf

Elboj, C. y Gràcia, S. (2005). La educación secundaria en comunidades de aprendizaje. El caso de Aragón. Educar, 35, 101-110. http://www.quadernsdigitals.net/datos_web/hemeroteca/r_73/nr_790/a 10614/10614.pdf

Elboj, C. y Niemelä, R. (2010). Sub-communities of Mutual Learners in the Classroom: The case of Interactive groups. Revista de Psicodidáctica, 15(2) 177-189. https://www.redalyc.org/pdf/175/17517246003.pdf

Elboj, C.; Espanya, M.; Flecha, R.; Imbernon, F.; Puigdellívol, I. y Valls, R. (1998). Comunidades de aprendizaje: sociedad de la información para todos (cambios sociales y algunas propuestas educativas). Contextos educativos, 1, 53-75.

https://publicaciones.unirioja.es/ojs/index.php/contextos/article/view/3 $71 / 0$

Elboj, C.; Puigdellívol, I.; Soler, M. y Valls, R. (2002). Comunidades de Aprendizaje: transformar la educación. Graó.

Ferrer, G. (2005). Hacia la excelencia educativa en las comunidades de aprendizaje: participación, interactividad y aprendizaje. Educar, 35, 61-70. https://educar.uab.cat/article/view/214

Flecha, R. y Soler, M. (2013). Turning difficulties into possibilities: engaging Roma families and students in school through dialogic learning. Cambridge Journal of Education, 43(4), 451-465. https://doi.org/10.1080/0305764X.2013.819068

Flecha, R. (2015). Sin diversidad no hay excelencia en el aprendizaje. Micro.es Diario Digital Ciudadano.

https://www.miciudadreal.es/2015/07/19/ramon-flecha-sin-diversidadno-hay-excelencia-en-el-aprendizaje/

García, R., Molina, S., Grande, L. A. y Buslon, N. (2016). Análisis de las interacciones entre alumnado y diversas personas adultas en actuaciones educativas de éxito: hacia la inclusión de todos y todas. Revista Latinoamericana de Educación Inclusiva, 10(1), 115-132. https://dialnet.unirioja.es/servlet/articulo?codigo $=1173790$ 
García, R., y Díez-Palomar, J. (2015). Learning communities: Pathways for educational success and social transformation through interactive groups in mathematics. European Educational Research Journal, 14(2) 151-166. https://doi.org/10.1177/1474904115571793

García, C.; Lastikka, A.L. y Petreñas, C. (2013). Comunidades de Aprendizaje. Scripta Nova. Revista Electrónica De Geografía y Ciencias Sociales, 427(7). http://www.ub.es/geocrit/sn/sn-427/sn427-7.htm

González, N., y Moll, L. (2002). Cruzando el puente: Building bridges to funds of knowledge. Journal of Educational Policy, 16(4), 623-641. https://doi.org/10.1177/0895904802016004009

Habermas, J. (1987). Teoría de la Acción Comunicativa. Taurus.

Herrero, C. y Brown, M. (2010). Distributed Cognition in CommunityBased Education. Revista de Psicodidáctica, 15(2), 253-268. https://www.ehu.eus/ojs/index.php/psicodidactica/article/view/816/68 8

INCLUD-ED Consortium (2011). Actuaciones de éxito en las escuelas europeas. Ministerio de Educación IFIIE.

http://orientacion.catedu.es/wp-content/uploads/2014/10/actuacionesde-exito-en-las-escuelas-europeas.pdf

Jasis, P. M. y Ordoñez-Jasis, R. (2012). Latino parent involvement. Examining commitment and empowerment in schools. Urban Education, 47(1), 65-89. https://doi.org/10.1177/0042085911416013

Jeynes, W. H. (2003). A meta-analysis. The Effects of Parental Involvement on Minority Children's Academic Achievement. Education and Urban Society, 35(2), 202-218.

http://citeseerx.ist.psu.edu/viewdoc/download?doi=10.1.1.875.3321yr ep=replytype $=$ pdf

Mercer, N., y Howe, C. (2012). Explaining the dialogic processes of teaching and learning: The value and potential of sociocultural theory. Learning, Culture and Social Interaction, 1(1), 12-21. http://dx.doi.org/10.1016/j.lcsi.2012.03.001

Molina, S. (2011). Los grupos interactivos: una práctica de las Comunidades de Aprendizaje para la inclusión del alumnado con discapacidad (Tesis doctoral). Universidad de Barcelona. 


\section{García López, López Fernández, \& Sánchez Pérez}

http://diposit.ub.edu/dspace/bitstream/2445/43112/2/SMR_TESIS.pdf Muntaner, J. J., Pinya, C., y De la Iglesia, B. (2015). Evaluación de los grupos interactivos desde el paradigma de la educación inclusiva. Revista Electrónica Interuniversitaria de Formación del Profesorado, 18(1), 141-159. https://doi.org/10.6018/reifop.18.1.214371

Nettles, S. M., y Greenberg, B. (1990). Community Involvement and Disadvantaged Students: A Review and Annotated Bibliography. The Johns Hopkins University. http://www.eric.ed.gov/PDFS/ED325601.pdf

Oliver, E., y De Gràcia, S. (2004). Grupos interactivos en Secundaria. Cuadernos de Pedagogía, 341, 70-72. https://revistas.comillas.edu/index.php/padresymaestros/article/downl $\mathrm{oad} / 5450 / 5263 /$

Oliver, E., y Gatt, S. (2010). From power-related communicative acts to dialogic communicative acts in classrooms organised in interactive groups. Revista Signos, 43, 279-294.

https://www.comunidaddeaprendizaje.com.es/uploads/materials/217/a 50277f722a8b812df72e70a0e6276cf.pdf

Prados, M. M., y Aguilera, A. (2013). Los alumnos y alumnas de la Universidad de Sevilla como voluntarios en Comunidades de Aprendizaje. Revista Andalucía Educativa, 79.

http://hdl.handle.net/11441/31140

Pulido, C., Elboj, C., Campdepadrós, R., y Cabré, J. (2014). Exclusionary and transformative dimensions: Communicative analysis enhancing solidarity among women to overcome gender violence. Qualitative Inquiry, 20(7), 889-894. https://doi.org/10.1177/1077800414537212

Racionero, S., García, R., Aubert, A., y Puigvert, L. (2009). Los modelos sociales de enseñanza: Los grupos interactivos. Competencias Básicas y Modelos de Enseñanza. Escuela, 4.

http://comclave.educarex.es/pluginfile.php/785/mod_folder/content/0/ Suplemento\%204.\%20Los\%20modelos\%20sociales\%20de\%20ense\% C3\%B1anza_los\%20grupos\%20interactivos.pdf?forcedownload=1

Racionero, S., Ortega, S., García, R. y Flecha, R. (2012). Aprendiendo Contigo. Hipatia Editorial.

Sánchez, M. (1999). Voices Inside Schools. La Verneda-Sant Martí: A 
School Where People Dare to Dream. Harvard Educational Review, 69(3), 320-335.

https://doi.org/10.17763/haer.69.3.gx588q10614q3831

Sánchez, M.C. y García, J.(coord.) (2019). Análisis de la situación de los

Centros Educativos de Comunidades de Aprendizaje en Castilla-La

Mancha. Curso 2017-2018. Ediciones de la UCLM.

https://ruidera.uclm.es/xmlui/bitstream/handle/10578/21198/Informe_

Final_Eval_Centros_CdA.pdf? sequence=1 yisAllowed=y

Sheldon, S. B. (2003). Linking School-Family-Community Partnerships in

Urban Elementary Schools to Student Achievement on State Tests.

The Urban Review, 35(2), 149-165.

https://doi.org/10.1023/A:1023713829693

Sordé, T., Serradell, O., Puigvert, L y Munté, A. (2014). Solidarity

Networks that Challenge Racialized Discourses: The Case of Romani

Immigrant Women in Spain. European Journal of Women's Studies,

21(1), 87-102. https://doi.org/10.1177/1350506813510425

Valls, M., Siles, G. y Molina, S. (2011). Formas de agrupación del

alumnado y su relación con el éxito escolar: Mixture, Streaming e

Inclusión. XII Congreso Internacional de Teoría de la Educación,

Universidad de Barcelona.

http://www.cite2011.com/Comunicaciones/Escuela/167.pdf

Valls, R., Buslón, N. y López, I. (2016). Grupos interactivos. Interacciones

que aumentan el aprendizaje y la solidaridad. Revista Padres y

Maestros, 367, 17-21. https://doi.org/10.14422/pym.i367.y2016.003

Valls, R., y Kyriakides, L. (2013). The power of interactive groups: how diversity of adults volunteering in classroom groups can promote inclusion and success for children of vulnerable minority ethnic populations. Cambridge Journal of Education, 43(1), 17-33.

http://doir.org/10.1080/0305764X.2012.749213

Vieira, L. y Puigdellívol, I. (2013). ¿Voluntarios dentro del aula? El rol del voluntariado en Comunidades de Aprendizaje. REXE. Revista de Estudios y Experiencias en Educación, 12(4), 37-55.

http://www.redalyc.org/pdf/2431/243129663003.pdf

Wanat, C. L. (2010). Challenges Balancing Collaboration and Independence: Analysis of Parents' Perceptions in One District. School Community 
56 García López, López Fernández, \& Sánchez Pérez

Journal, 20(1), 159-186. http://files.eric.ed.gov/fulltext/EJ891837.pdf

Juan García López is member of the Subred Universitaria de Comunidades de Aprendizaje de Castilla-La Mancha, Spain

Sandra López Fernández is a Professor at the Universidad de Castilla-La Mancha, Spain

M Carmen Sánchez Pérez is a Professor at the Universidad de Castilla-La Mancha, Spain

Contact Address: suca.clm@uclm.es 